\title{
Adsorption energies of benzene on close packed transition metal surfaces using the random phase approximation
}

\author{
José A. Garrido Torres, ${ }^{1}$ Benjamin Ramberger, ${ }^{2}$ Herbert A. Früchtl, ${ }^{1}$ Renald Schaub, ${ }^{1}$ and Georg Kresse ${ }^{2, *}$ \\ ${ }^{1}$ EaStCHEM and School of Chemistry, University of St Andrews, KY16 9ST, St Andrews, UK \\ ${ }^{2}$ Faculty of Physics and Center for Computational Materials Sciences, \\ University of Vienna, Sensengasse 8/12, 1090 Wien, AT
}

(Dated: October 11, 2017)

\begin{abstract}
The adsorption energy of benzene on various metal substrates is predicted using the random phase approximation (RPA) for the correlation energy. Agreement with available experimental data is systematically better than $10 \%$ for both coinage and reactive metals. The results are also compared with more approximate methods, including vdW-density functional theory (DFT), as well as dispersion corrected DFT functionals. Although dispersion corrected DFT can yield accurate results, for instance, on coinage metals, the adsorption energies are clearly overestimated on more reactive transition metals. Furthermore, coverage dependent adsorption energies are well described by the RPA. This shows that for the description of aromatic molecules on metal surfaces further improvements in density functionals are necessary, or more involved many body methods such as the RPA are required.
\end{abstract}

The accurate prediction of adsorption energies of molecules on metal surfaces is a challenging subject in condensed matter physics, physical chemistry, as well as applied catalysis research. It is now well understood that although semi-local functionals generally predict trends between different metal surfaces reasonably well, absolute adsorption energies even for prototypical molecules can be inaccurate [1-4]. This is particularly true for the very difficult cases of aromatic molecules, since their adsorption involves a mixture of covalent bonding and van der Waals (vdW) bonding. The former is overestimated by the most commonly used DFT functional, the PerdewBurke-Ernzerhof (PBE) functional [5], whereas the later is not captured at all by gradient corrected functionals. Sometimes these two errors compensate, but most often this is not the case. Progress in the inclusion of vdW corrections has been remarkable in the last years. The most successful schemes are additive D3 dispersion corrections by Grimme and coworkers [6, 7], the similar scheme of Tkatchenko and Scheffler (TS) [8], as well as van der Waals density functionals (vdW-DF) that depend on the density at two positions in space. The later were originally introduced by Dion, Lundqvist and coworkers [9], but since the accuracy of the first functional was not always satisfactory, many "improved" functionals emerged soon after (e.g. Ref. 10-12).

The adsorption of aromatic molecules has been thoroughly investigated using different functionals on an extensive range of (111) transition metal surfaces, including coinage and catalytic substrates [13-19]. The results including vdW corrections are overall quite promising, however, a careful inspection of the numbers shows that they are not entirely satisfactory. In particular, on catalytic substrates, the adsorption energies for aromatic molecules tend to be overestimated. It is a simple matter to understand this issue. In the approximate methods described above, either a polarizability is assigned to individual atoms yielding predominantly pair-wise interactions between any two atoms, or a vdW like interaction between any two points in space is introduced using the jellium gas as reference. The atom based partitioning neglects that in metal substrates, local fluctuations by $d$ electrons are strongly screened by the electrons at the Fermi-surface (Drude term). On the other hand, the jellium electron gas is not necessarily an accurate reference able to describe the localized $d$ electron fluctuations. Thus the interplay between local polarizability and metallic screening is not captured by either of the two approximations. Likewise, both methods are combined with approximate density functionals that sometimes fail to describe covalent bonding contributions accurately, as for instance amply reported for $\mathrm{CO}$ on metal surfaces $[4,20,21]$.

The only seamless approach capable of describing local $d$ electron fluctuations, free electron like excitations across the Fermi-level, as well as covalent bonding, is the random phase approximation to the correlation energy [22-26]. In this approximation, first a DFT calculation is performed using an approximate density functional (here the PBE functional [5]). Then the exact exchange and RPA correlation energy are evaluated as [23, 27-32]:

$$
E=E_{\mathrm{EXX}}+\underbrace{\frac{1}{2 \pi} \int_{0}^{\infty} \mathrm{d} \nu \operatorname{Tr}[\ln (1-\chi(\mathrm{i} \nu) \mathrm{v})+\chi(\mathrm{i} \nu) \mathrm{v}]}_{E_{\mathrm{RPA}}},
$$

where $E_{\mathrm{EXx}}$ is the Hartree-Fock energy functional determined using PBE orbitals, $\chi(\mathrm{i} \nu)$ is the independent particle polarizability calculated using PBE orbitals and one electron energies, and $\mathrm{v}$ is the Coulomb kernel. Here, we use the RPA to evaluate the adsorption energy of benzene on various substrates. We show that only RPA yields reference quality results in excellent agreement with experiment.

LEED and STM experiments as well as theory show 


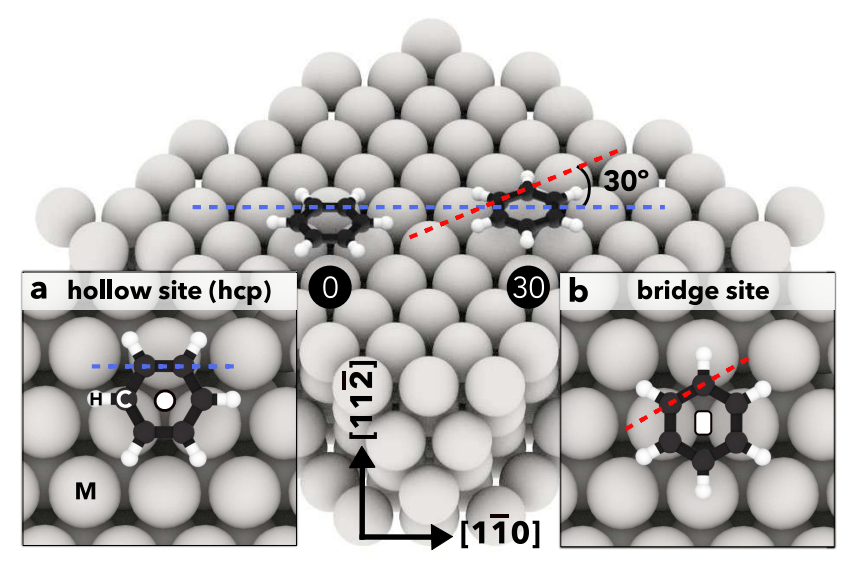

Figure 1. (color online) Representation of the two most common adsorption sites for benzene on transition metals. Magnifications from a top-view perspective for benzene adsorbed on the (a) 3-fold hcp and (b) 2-fold bridge sites. The blue and red dashed-lines are set to emphasise the different alignment of the $\mathrm{C}-\mathrm{C}$ bonds for the 0 and 30 degrees configurations. Carbon, hydrogen and metal atoms are represented as black, white and grey spheres, respectively.

that the benzene molecule adsorbs mainly at two sites, the 2 -fold (bridge) or the 3 -fold (hollow) site with the $\mathrm{C}_{6}$ ring parallel to the surface plane [17, 18, 33-39] (see Fig. 1 ). The preference for one or the other site is driven by a combination of different factors, such as the nature of the substrate [35], the coverage [36] or the presence of coadsorbates [38]. For coinage metals, the carbon-hydrogen planarity is not disturbed [40], whereas the molecule is significantly deformed when strong chemisorption occurs [41].

Our strategy to calculate the adsorption energy is to fully relax the benzene molecule for each of the considered functionals for both adsorption registries (hcp, bridge). Although, we have recently presented a method to calculate forces between the atoms in the RPA [42], for efficiency reasons we have decided to use PBE geometries for the RPA calculations in the present work. The calculations were performed using the cubic scaling RPA of Kaltak and coworkers [43]. For $\mathrm{Cu}, \mathrm{Ag}$ and $\mathrm{Au}$, we found that a slightly more refined procedure was necessary to obtain accurate adsorption energies. For these coinage metals, we varied the distance between the center of mass of the molecule and the substrate, relaxed all other degrees of freedom using PBE, and then calculated the RPA energies for all considered moleculesurface distances. This procedure was required, since none of the functionals yielded a satisfactory surfacemolecule distance for benzene on these metals. The optimized substrate-molecule distance for RPA was $\sim 3.0 \AA$, $2.95 \AA$ and $3.02 \AA$ for $\mathrm{Cu}, \mathrm{Ag}$, and $\mathrm{Au}$, respectively. As an example, results for the energy-versus distance curve for $\mathrm{Au}$ are shown in Fig. 2.
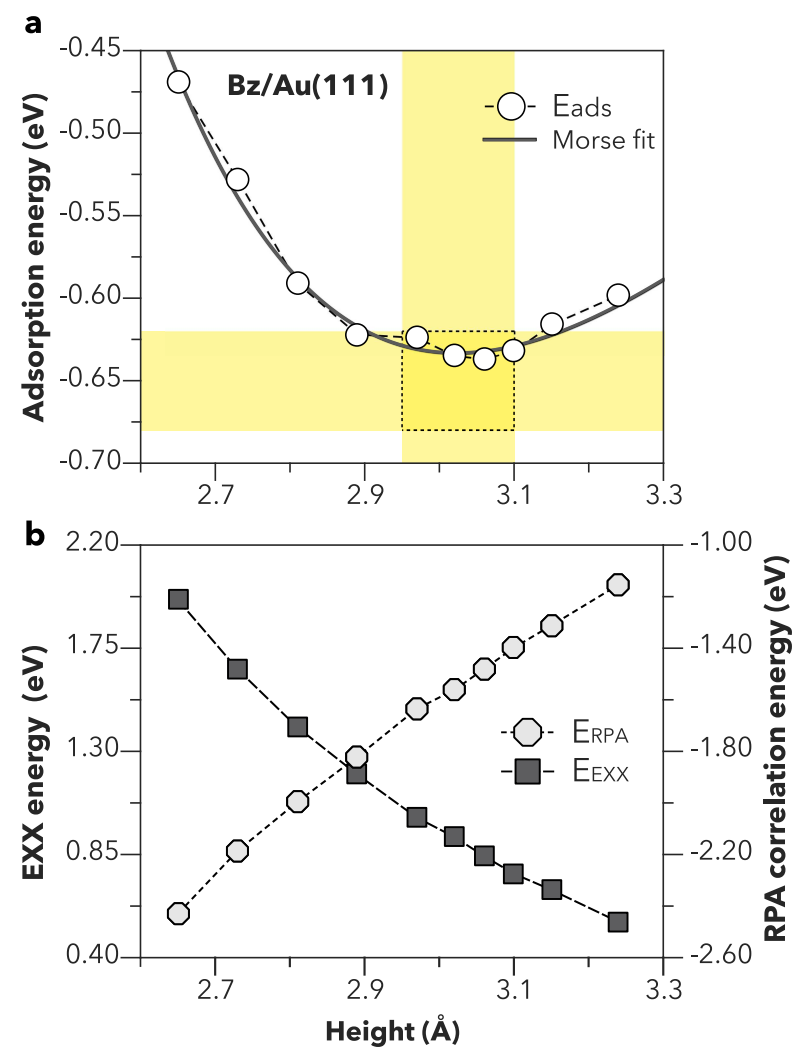

Figure 2. (color online) (a) Plot of adsorption energy versus benzene height when adsorbed on $\mathrm{Au}(111)$. The roughness is related to the use of a fairly coarse grid for the fast Fourier transformations in the RPA total energy calculations. The yellow rectangles mark the range of experimental values with uncertainties $[16,39]$. The minimum of the energy curve lies within the experimental range (highlighted by the dashed rectangle) (b) Individual $E_{\mathrm{EXX}}$ and $E_{\mathrm{RPA}}$ contributions as a function of distance. Notice the large compensation between the two terms.

We start with a brief discussion of the DFT results. The coinage metals are characterized by a completely filled $d$ shell, and adsorption is essentially dominated by $\mathrm{vdW}$ contributions. Since the PBE functional does not account for $\mathrm{vdW}$ interactions, the predicted adsorption energies on the coinage metals are smaller than $0.1 \mathrm{eV}$ for PBE. Furthermore, the predicted surface-molecule distances are typically about $3.8 \AA$, which are clearly much larger than the values obtained from the RPA (compare also Fig. 2). On the open shell $d$ metals (Ni, Pd, Pt and $\mathrm{Rh})$, adsorption energies of about $1.0 \mathrm{eV}$ are predicted, which are about $0.8 \mathrm{eV}$ smaller than the experimental values. The atom-based vdW correction schemes (PBE-D3 [6] and PBE-TS [8]) behave remarkably similar, with corrections to $\mathrm{PBE}$ of $0.7 \mathrm{eV}$ for coinage metals and $1.2 \mathrm{eV}$ for the considered reactive metals. The Ni substrate is a clear outliner, with corrections amounting to $2 \mathrm{eV}$. This indicates that there are issues with the parametrization of $\mathrm{vdW}$ corrections for magnetic $\mathrm{Ni}$, and possibly mag- 
netic transition metals in general. The optB86b-vdW functional also yields reasonable results, with corrections of $0.6 \mathrm{eV}$ for coinage metals, and $1.25 \mathrm{eV}$ for the reactive metals. The values are seemingly somewhat more consistent than for the PBE-D3 and PBE-TS cases. In particular, for benzene adsorption on $\mathrm{Ni}$, the corrections are now in line with the other transition metals. Results for the vdW-DF [9] and vdW-DF2 [10] functionals are disappointing. The adsorption energies behave in a very non-systematic manner. Even, if we exclude the magnetic Ni (neither of the two functionals are suitable for magnetic substrates [44]), meaningful improvements compared to PBE can only be observed for the coinage metals.

Table I. Calculated adsorption energies (in eV) for benzene adsorbed on different (111) metal substrates at medium coverage. Experimental values from the literature are included for comparison (in bold are the theoretical values that are within the experimental errors). Calculations were performed for a $(2 \sqrt{3} \times 4)$ unit cell with 4 layers. The topmost 2 layers were relaxed. $3 \times 3 \times 1 k$-points were used for the RPA, and corrected for $\mathrm{k}$-point errors using the PBE functional. The asterisk * marks those cases where the bridge configuration is energetically more favourable than hcp.

\begin{tabular}{lccccccc}
\hline \hline & $\mathrm{Cu}$ & $\mathrm{Ag}$ & $\mathrm{Au}$ & $\mathrm{Ni}$ & $\mathrm{Pd}$ & $\mathrm{Pt}$ & $\mathrm{Rh}$ \\
\hline PBE & $0.09^{*}$ & $0.05^{*}$ & 0.05 & $1.04^{*}$ & $1.16^{*}$ & $0.82^{*}$ & 1.46 \\
PBE-D3 & 0.98 & 0.78 & 0.83 & 3.09 & $2.34^{*}$ & $2.09^{*}$ & 2.58 \\
PBE-TS & 0.87 & 0.77 & 0.80 & $2.85^{*}$ & $2.10^{*}$ & $1.99^{*}$ & $2.72^{*}$ \\
vdW-DF & 0.53 & $0.46^{*}$ & 0.53 & 0.56 & $0.83^{*}$ & $0.37^{*}$ & 1.16 \\
vdW-DF2 & 0.75 & 0.43 & $0.43^{*}$ & -0.07 & $0.63^{*}$ & $1.33^{*}$ & 0.63 \\
optB86b-vdW & $\mathbf{0 . 6 9}$ & $\mathbf{0 . 6 9}$ & $0.76^{*}$ & $2.25^{*}$ & $2.41^{*}$ & $2.12^{*}$ & 2.68 \\
RPA & $\mathbf{0 . 6 6}$ & $\mathbf{0 . 6 3}$ & $\mathbf{0 . 6 4}$ & $1.46^{*}$ & $\mathbf{1 . 7 2}^{*}$ & $\mathbf{1 . 7 4}^{*}$ & 2.08 \\
\hline Exp. & $0.69^{a}$ & $0.68^{a}$ & $0.65^{a}$ & - & $1.74^{b}$ & $1.67^{c}$ & - \\
$\Delta$ Exp. & \pm 0.04 & \pm 0.05 & \pm 0.03 & - & \pm 0.30 & \pm 0.17 & - \\
\hline \hline
\end{tabular}

${ }^{a}$ Ref. [39]; recent interpretation of TPD spectra based on the Polanyi-Wigner equation [45].

${ }^{b}$ Ref. [17]; TPD experiments using the Redhead analysis [46].

${ }^{c}$ Ref. [47]; microcalorimetry measurements for benzene at $\theta=0.80 \mathrm{ML}$, corresponding to the $2 \sqrt{3} \times 4$ unit cell.

The best agreement with experiment is obtained for the RPA, in which every single predicted value falls within the experimental error bars (highlighted in bold in Table I). The low uncertainty values of the experimental adsorption energies for the coinage metals (errors bars below $0.05 \mathrm{eV}$ ) can be used to assess the accuracy of the RPA method. On the other hand, for the metals in which the experimental error bars are large (e.g. Pd and Pt), the RPA calculated values and the experimental values seem to reinforce each other. This suggests that the RPA can be used as a predictive tool for assessing the quality of vdW correction schemes and it is especially useful if no conclusive statement can be made solely from experimental values due to large error bars. RPA
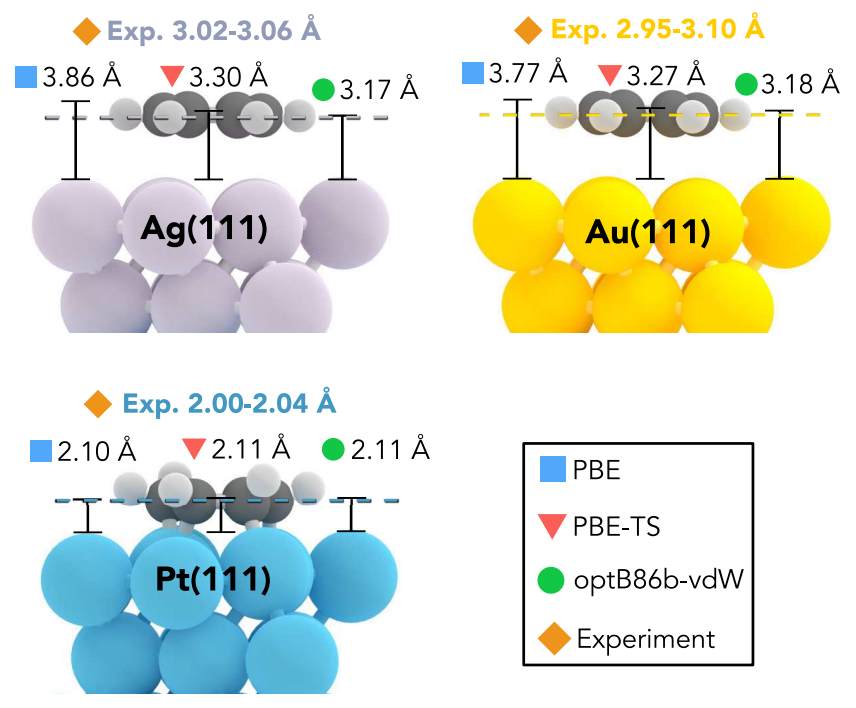

Figure 3. (color online) Averaged carbon-metal distances for benzene on $\mathrm{Ag}, \mathrm{Au}$ and $\mathrm{Pt}$ using the PBE, PBE-TS and optB86b-vdW functionals. Experimental heights are included for comparison $\mathrm{Ag}$ [39], $\mathrm{Au}[16,39]$ and $\mathrm{Pt}$ [47, 51].

suggests that PBE-D3, PBE-TS and optB86b-vdW overestimate the vdW corrections slightly for coinage metals and substantially for reactive metals. By this statement we mean that the correction (the difference between PBE and PBE-D3) is up to twice as large as the difference between RPA and PBE. But this error is also nonsystematic: for $\mathrm{Cu}, \mathrm{Ag}, \mathrm{Au}$, and $\mathrm{Pt}$, the error seems to be only about $25 \%$, however it increases to almost $100 \%$ for $\mathrm{Pd}$ and $\mathrm{Rh}$. It is not unlikely that a substantial part of the error is already present in the parent DFT functional. But it is equally well possible that the error is related to the neglect of the interplay between the local excitations and jellium like screening in metals. If one neglects the metallic screening, the interaction between the substrate atoms and the molecule is expected to be overestimated [48, 49]. Inclusion of this effect slightly improves agreement with experiment, but still yields too large adsorption energies (Pd $2.14 \mathrm{eV}, \mathrm{Rh} 2.52 \mathrm{eV})$ [16]. Many body dispersion corrections [50] provide a more systematic approach to resolve this issue. However, adsorption to metals is not straightforward [52], and even then the issue of the accuracy of the underlying semi-local DFT functional remains to be addressed.

We now comment briefly on the geometries. To this end we show in Fig. 3 results for $\mathrm{Ag}, \mathrm{Au}$, and $\mathrm{Pt}$, for which experimental geometries are accurately known. It is clear that PBE predicts a much too large benzenesubstrate distance on $\mathrm{Ag}$ and $\mathrm{Au}$, but even the optB86b$\mathrm{vdW}$ functional yields distances that are somewhat larger than experimentally measured. The value of $2.95 \AA$ $(3.02 \AA)$ obtained with the RPA for $\mathrm{Ag}(\mathrm{Au})$ is very close to the experimental range of values. As an example for strong chemisorption, we also show the results 
for $\mathrm{Pt}$, where we observe that the different functionals yield very similar benzene substrate distances. We also compared RPA adsorption energies determined using different geometries, for instance the optB86b-vdW geometries, and found little variations for the energies between the geometries. Furthermore, an optimization of the surface-benzene distance for $\mathrm{Pt}$, in the same manner as for the coinage metals, changed the results very little. This is an a posteriori justification for our choice to use PBE geometries for the RPA calculations for reactive substrates. In summary, for coinage metals the geometries change substantially if vdW contributions are taken into account and only the RPA yields entirely satisfactory results, whereas we see little height variations between functionals for reactive metals.

The final issue we would like to address in this letter is whether the RPA also improves the description of the coverage dependence of the adsorption energy. Microcalorimetry experiments have been performed by the group of Campbell [47] to investigate this dependence for benzene on $\mathrm{Pt}(111)$. Panel (b) in Fig. 4 shows the results for all functionals, including PBE, vdW-DF and vdW-DF2. As before, results for these three functionals are not satisfactory on an absolute scale. We therefore disregard them in further discussion and concentrate on the results for the more accurate functionals in panel (c). The RPA shows a steady decrease of the average adsorption energy (corresponding to the integral of the differential heat of adsorption). This is expected, because of repulsion between the molecules as well as a progressive passivation of the substrate atoms [2]. An issue that all density functionals share is that structure (II) is unstable, since it lies above the connecting line between phase (I) and (III). Interestingly, the RPA predicts this phase to be stable. It is clear that RPA matches the experimental coverage dependence very well, falling always within the experimental error bars. But we note that the error in the vdW corrected functionals is mostly in the absolute value of the adsorption energy and less so in the repulsive interaction between the molecules.

In summary, we have calculated the adsorption energy of benzene on coinage metals as well as prototypical catalytic substrates. Agreement with the available experimental data for adsorption energies is excellent, both at the lowest considered coverage and at a range of coverages for Pt. Furthermore, for coinage metals the RPA predicts benzene-substrate distances of about $3.0 \AA$ in perfect agreement with experiment, significantly smaller than for semi-local functionals and somewhat smaller than for most vdW corrected functionals (3.2 $\AA$ ). Although our RPA calculations are $\sim 50$ times more expensive than PBE, they are very affordable: on 192 Intel Xeon v2 cores one energy calculation required typically two to three hours. Hence reference results for adsorption on metal surfaces can now be easily and reliably obtained even for complex molecules with mixed covalent and van a
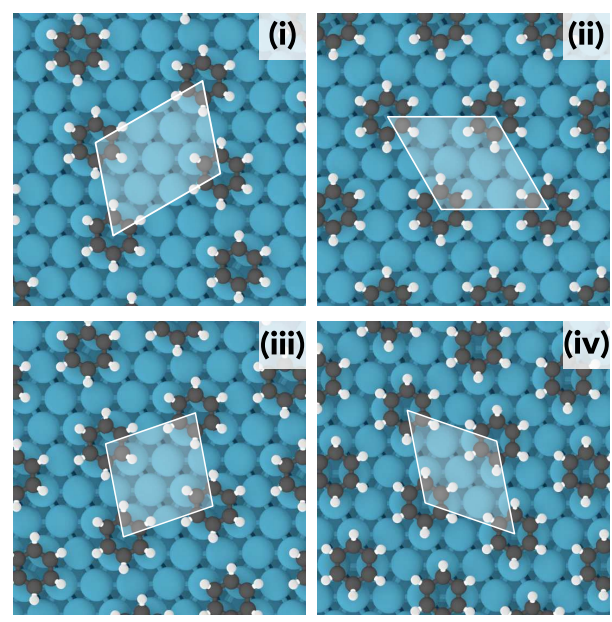

b
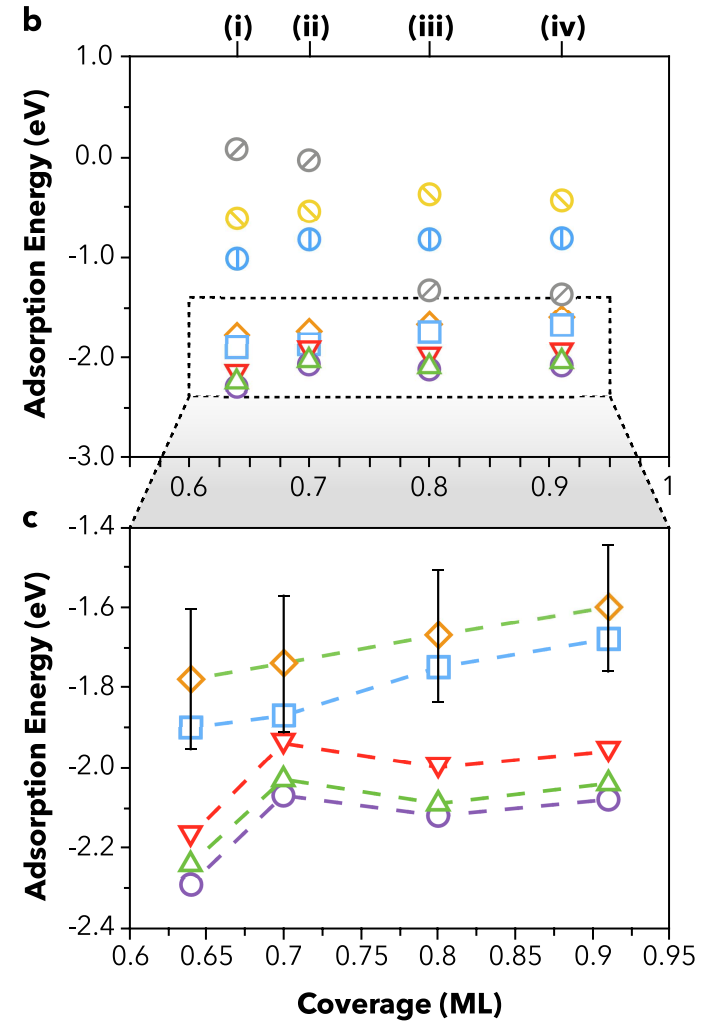

\begin{tabular}{|llll|}
\hline (1) PBE & $\triangle$ PBE-D3 & $\nabla$ PBE-TS & $\diamond$ vdW-DF \\
$\oslash$ vdW-DF2 & O optB86b-vdW & $\square$ RPA & $\diamond$ Exp. \\
\hline
\end{tabular}

Figure 4. (color online) Adsorption energy as function of the coverage for benzene on $\operatorname{Pt}(111)$. (a) Considered geometries for benzene at coverages $\theta_{\mathrm{i}}=0.64, \theta_{\mathrm{ii}}=0.71, \theta_{\mathrm{iii}}=0.80$ and $\theta_{\mathrm{iv}}=0.91$ monolayers (ML), defined by the corresponding surface unit cells: (i) $=\left(\begin{array}{lllll}4 & 2 & \mid & -1 & 3\end{array}\right),($ ii $)=\left(\begin{array}{lllll}3 & 0 & \mid & 0 & 3\end{array}\right)$, $($ iii $)=\left(\begin{array}{lllll}3 & 1 & \mid & 1 & 3\end{array}\right)$ and (iv) $=\left(\begin{array}{lllll}2 & -1 & \mid & 1 & 3\end{array}\right)$. (b) Adsorption energy (in eV) for benzene on $\mathrm{Pt}(111)$ for those coverages (in ML). (c) Magnification of the previous plot in the area near the experimental energy range (dotted lines are added to underline the trends). Integral of the differential heat of adsorption and error bars, corresponding to the spread of the experimental results, are included from Ref. [47]. For the coverage, we have adopted the convention of Ihm and coworkers [47]. 
der Waals bonding. This is an important step towards an accurate first principles modelling of substrate-adsorbate interactions. The present study establishes that a similar systematic improvement as for $\mathrm{CO}$ on metal surfaces [25] carries over to the much more challenging aromatic molecules.

Acknowledgment: We acknowledge financial support from the Scottish Funding Council (through EaStCHEM and SRD-Grant HR07003) and from EPSRC (PhD studentship for JAGT, EP/M506631/1). Funding by the Austrian Science Fund (FWF): F41 (SFB ViCoM) is grateful acknowledged. Computations were predominantly performed on the Vienna Scientific Cluster VSC3. The research data supporting this publication can be accessed at: http://dx.doi.org/10.17630/dd2bd186-f2bc4483-93c6-d7288862c097

* georg.kresse@univie.ac.at

[1] B. Hammer and J. K. Nørskov, Nature 376, 238 (1995).

[2] J. K. Nørskov, T. Bligaard, B. Hvolbæk, F. AbildPedersen, I. Chorkendorff, and C. H. Christensen, Chem. Soc. Rev. 37, 2163 (2008).

[3] J. K. Nørskov, T. Bligaard, J. Rossmeisl, and C. H. Christensen, Nat. Chem. 1, 37 (2009).

[4] P. J. Feibelman, B. Hammer, J. K. Nørskov, F. Wagner, M. Scheffler, R. Stumpf, R. Watwe, and J. Dumesic, J. Phys. Chem. B 105, 4018 (2001).

[5] J. P. Perdew, K. Burke, and M. Ernzerhof, Phys. Rev. Lett. 77, 3865 (1996).

[6] S. Grimme, J. Antony, S. Ehrlich, and H. Krieg, J. Chem. Phys. 132, 154104 (2010).

[7] S. Grimme, S. Ehrlich, and L. Goerigk, J. Comput. Chem. 32, 1456 (2011).

[8] A. Tkatchenko and M. Scheffler, Phys. Rev. Lett. 102, 073005 (2009).

[9] M. Dion, H. Rydberg, E. Schröder, D. C. Langreth, and B. I. Lundqvist, Phys. Rev. Lett. 92, 246401 (2004).

[10] E. D. Murray, K. Lee, and D. C. Langreth, J. Chem. Theory Comput. 5, 2754 (2009).

[11] J. Klimeš and A. Michaelides, J. Chem. Phys. 137, 120901 (2012).

[12] K. Berland, V. R. Cooper, K. Lee, E. Schröder, T. Thonhauser, P. Hyldgaard, and B. I. Lundqvist, Rep. Prog. Phys. 78, 066501 (2004).

[13] S. Yamagishi, S. J. Jenkins, and D. A. King, J. Chem. Phys. 114, 5765 (2001).

[14] C. Morin, D. Simon, and P. Sautet, J. Phys. Chem. B 108, 5653 (2004).

[15] K. Toyoda, I. Hamada, S. Yanagisawa, and Y. Morikawa, J. Nanosci. Nanotechnol. 11, 2836 (2011).

[16] W. Liu, J. Carrasco, B. Santra, A. Michaelides, M. Scheffler, and A. Tkatchenko, Phys. Rev. B 86, 245405 (2012).

[17] H. Yildirim, T. Greber, and A. Kara, J. Phys. Chem. C 117, 20572 (2013).

[18] J. Carrasco, W. Liu, A. Michaelides, and A. Tkatchenko, J. Chem. Phys. 140, 084704 (2014).

[19] D. P. Miller, S. Simpson, N. Tymińska, and E. Zurek, J. Chem. Phys. 142, 101924 (2015).
[20] L. Köhler and G. Kresse, Phys. Rev. B 70, 165405 (2004).

[21] A. Stroppa and G. Kresse, New J. Phys. 10, 063020 (2008).

[22] M. Rohlfing and T. Bredow, Phys. Rev. Lett. 101, 266106 (2008).

[23] J. Harl and G. Kresse, Phys. Rev. Lett. 103, 056401 (2009).

[24] S. Lebègue, J. Harl, T. Gould, J. G. Ángyán, G. Kresse, and J. F. Dobson, Phys. Rev. Lett. 105, 196401 (2010).

[25] L. Schimka, J. Harl, A. Stroppa, A. Grüneis, M. Marsman, F. Mittendorfer, and G. Kresse, Nat. Mater. 9, 741 (2010).

[26] T. Olsen, J. Yan, J. J. Mortensen, and K. S. Thygesen, Phys. Rev. Lett. 107, 156401 (2011).

[27] P. Nozières and D. Pines, Phys. Rev. 111, 442 (1958).

[28] D. C. Langreth and J. P. Perdew, Phys. Rev. B 15, 2884 (1977).

[29] T. Miyake, F. Aryasetiawan, T. Kotani, M. van Schilfgaarde, M. Usuda, and K. Terakura, Phys. Rev. B 66, 245103 (2002).

[30] M. Fuchs, Y.-M. Niquet, X. Gonze, and K. Burke, J. Chem. Phys. 122, 094116 (2005).

[31] F. Furche, J. Chem. Phys. 129, 114105 (2008).

[32] J. Harl, L. Schimka, and G. Kresse, Phys. Rev. B 81, 115126 (2010).

[33] M. Neuber, F. Schneider, C. Zubraegel, and M. Neumann, J. Phys. Chem. 99, 9160 (1995).

[34] M. A. Van Hove, R. F. Lin, and G. a. Somorjai, J. Am. Chem. Soc. 108, 2532 (1986).

[35] H. Ohtani, B. Bent, C. Mate, M. Van Hove, and G. Somorjai, Appl. Surf. Sci. 33-34, 254 (1988).

[36] O. Schaff, V. Fernandez, P. Hofmann, K.-M. Schindler, A. Theobald, V. Fritzsche, A. Bradshaw, R. Davis, and D. Woodruff, Surf. Sci. 348, 89 (1996).

[37] T. J. Rockey, M. Yang, and H.-L. Dai, J. Phys. Chem. B 110, 19973 (2006).

[38] G. Witte, H. Range, J. P. Toennies, and C. Wöll, Phys. Rev. Lett. 71, 1063 (1993).

[39] W. Liu, F. Maaß, M. Willenbockel, C. Bronner, M. Schulze, S. Soubatch, F. S. Tautz, P. Tegeder, and A. Tkatchenko, Phys. Rev. Lett. 115, 036104 (2015).

[40] D. Syomin, J. Kim, B. E. Koel, and G. B. Ellison, J. Phys. Chem. B 105, 8387 (2001).

[41] B. E. Koel, J. E. Crowell, C. M. Mate, and G. A. Somorjai, J. Phys. Chem. 88, 1988 (1984).

[42] B. Ramberger, T. Schäfer, and G. Kresse, Phys. Rev. Lett. 118, 106403 (2017).

[43] M. Kaltak, J. Klimeš, and G. Kresse, J. Chem. Theory Comput. 10, 2498 (2014).

[44] T. Thonhauser, S. Zuluaga, C. A. Arter, K. Berland, E. Schröder, and P. Hyldgaard, Phys. Rev. Lett. 115, 136402 (2015).

[45] D. A. King, Surf. Sci. 47, 384 (1975).

[46] P. A. Redhead, Vacuum 12, 203 (1962).

[47] H. Ihm, H. M. Ajo, J. M. Gottfried, P. Bera, and C. T. Campbell, J. Phys. Chem. B 108, 14627 (2004).

[48] J. Rehr, E. Zaremba, and W. Kohn, Phys. Rev. B 12, 2062 (1975).

[49] E. Zaremba and W. Kohn, Phys. Rev. B 13, 2270 (1976).

[50] A. Tkatchenko, R. A. DiStasio Jr, R. Car, and M. Scheffler, Phys. Rev. Lett. 108, 236402 (2012).

[51] A. Wander, G. Held, R. Hwang, G. Blackman, M. Xu, P. de Andres, M. Van Hove, and G. Somorjai, Surf. Sci. 
249, 21 (1991).

[52] V. G. Ruiz, W. Liu, E. Zojer, M. Scheffler, and A. Tkatchenko, Phys. Rev. Lett. 108, 146103 (2012). 